\title{
Taohong Siwu-Containing Serum Enhances Angiogenesis in Rat Aortic Endothelial Cells by Regulating the VHL/HIF-1 $\alpha /$ VEGF Signaling Pathway
}

\author{
Zhi Tang, ${ }^{1}$ Wangyang Li, ${ }^{2}$ Hongzan Xie, ${ }^{1}$ Shengping Jiang, ${ }^{1}$ Yunqing Pu, ${ }^{1}$ and Hui Xiong $\oplus^{2}$ \\ ${ }^{1}$ Department of Orthopedics, Xiangtan Chinese Medicine Hospital, Xiangtan 411100, China \\ ${ }^{2}$ Department of Clinical Medicine, Hunan University of Traditional Chinese Medicine, Changsha 410208, China \\ Correspondence should be addressed to Hui Xiong; xionghuihunan@163.com
}

Received 24 November 2020; Revised 27 July 2021; Accepted 1 October 2021; Published 22 November 2021

Academic Editor: Md. Sahab Uddin

Copyright (C) 2021 Zhi Tang et al. This is an open access article distributed under the Creative Commons Attribution License, which permits unrestricted use, distribution, and reproduction in any medium, provided the original work is properly cited.

Background. The incidence of bone fracture and bone-related diseases is increasing every year. Angiogenesis plays a vital role in fracture healing and bone repair. This study assessed the benefits of Taohong Siwu (TSW) decoction on angiogenesis in isolated rat aortic endothelial cells (RAEC) treated with TSW-containing serum. Methods. The components of TSW decoction were analyzed by liquid chromatography-mass spectrometry (LC-MS). TSW-containing serum was prepared by gavage of TSW decoction to Sprague-Dawley (SD) rats. The effects of TSW-containing serum on the viability, migration, wound healing, and angiogenesis of RAEC were detected by the MTT, transwell, wound healing, and Matrigel lumen formation assays, respectively. In addition, the effects of an HIF-1 $\alpha$ inhibitor on TSW-containing serum-induced RAEC were also assessed. The effects of TSW-containing serum on the expression of the HIF- $1 \alpha$ signaling pathway were evaluated by qRT-PCR and western blot analysis. Results. LC-MS revealed that TSW decoction primarily contained isomaltulose, choline, D-gluconic acid, L-pipecolic acid, hypotaurine, albiflorin, and tryptophan. TSW-containing serum significantly increased the viability, migration, wound healing, and angiogenesis of RAEC in a dose-dependent manner. Furthermore, our results demonstrated that HIF- $1 \alpha$ and VEGF expressions were increased in the cells of TSW-containing serum groups, whereas VHL expression was decreased. The effects of TSW-containing serum were reversed by treatment with an HIF- $1 \alpha$ inhibitor. Conclusion. These results suggested that TSW decoction enhanced angiogenesis by regulating the VHL/HIF-1 $\alpha$ /VEGF signaling pathway.

\section{Background}

The incidence of bone fracture and bone-related diseases is increasing every year. Fracture healing and bone repair are complicated pathological processes [1]. Patients with bonerelated diseases suffer from delayed healing following fracture treatment for some reasons, such as without proper vascularization and blood supply $[1,2]$. Several factors in the local microenvironment, such as oxygen saturation, $\mathrm{PH}$, cytokines, and growth factors, may significantly affect fracture healing via directly affecting blood vessel formation, proliferation and differentiation of bone cells, and the deposition of minerals [3]. Few studies on angiogenesis related to fracture healing and the development of related drugs are worthy of in-depth research.
Rat aortic endothelial cells (RAEC) were isolated from Sprague-Dawley (SD) rats, and then RAEC were identified and cultured. RAEC are commonly used to study cardiovascular diseases [4]. Wound healing and tissue regeneration are closely related to forming a sufficient blood vessel network [5]. The endothelial cell tubule formation could act as a marker of angiogenesis, which is also a prerequisite and basis for bone healing [6]. Proper angiogenesis is indispensable for fracture healing, so RAEC are also used to study fracture healing, bone tissue engineering, and so on [2]. In this study, RAEC were used to study the development of auxiliary drugs related to fracture healing.

Fractures are usually accompanied by the rupture and embolization of blood vessels, which causes local and systemic hemorheological changes, increasing blood viscosity, 
slowing local microcirculation blood flow, and decreasing tissue oxygen saturation [1]. Hypoxia might initiate the compensatory response and the transcription of hypoxia response genes (HRG) in the cell, such as vascular endothelial growth factor (VEGF), erythropoietin (EPO), tyrosine hydroxylase, and several enzymes involved in glycolysis $[7,8]$. Studies have shown that such a response is dependent upon hypoxia-inducible factor- $1 \alpha$ (HIF-1 $\alpha)$ [9, 10]. Under hypoxic conditions, HIF- $1 \alpha$ is upregulated as a central regulator of angiogenesis $[11,12]$. It directly participates in the entire process of angiogenesis by altering the expression of related growth factors [9, 12-14]. In addition, Hippel-Lindau (VHL) has been shown to inhibit the accumulation of HIF-1 $\alpha[15,16]$.

It is well known that many traditional Chinese medicines (TCM) promote blood circulation and remove blood stasis. They are beneficial to the local and systemic blood circulation during the early stages of fracture healing, which may improve local blood oxygen status and create a good internal environment for fracture healing [17-20]. Taohong Siwu (TSW) decoction is one of the most popular TCM for promoting blood circulation, removing blood stasis, and treating orthopedic diseases [21, 22]. Previous studies have shown that TSW-containing serum enhances VEGF expression in human endothelial cells through the PI3K/AkteNOS axis [23]. TSW decoction promotes angiogenesis during early pregnancy in rats by increasing the expression of Ang-1/2 and Tie-2 [24]. However, the mechanism of TSW decoction on the regeneration of blood vessels and fracture healing remains unclear. In this study, we analyzed the components of TSW decoction and investigated the possible mechanism for its effects on angiogenesis.

\section{Material and Methods}

2.1. Animals and Breeding Environment. Twenty male SD rats weighing 200-260 g were purchased from the Hunan Slack Jingda Experimental Animal Co. Ltd. (license \# SCXK (Hunan) 2013-0004). The rats were raised at the Experimental Animal Center of the Hunan University of Traditional Chinese Medicine. They were housed in a standard animal room at a temperature of $25^{\circ} \mathrm{C} \pm 2{ }^{\circ} \mathrm{C}$ and $50 \% \pm 5 \%$ humidity with $12 \mathrm{~h}$ of light and dark cycle. The cages were cleaned once a week, and the litter was replaced as needed. Animals were provided free access to water and food provided by the Experimental Animal Center of the Hunan University of Traditional Chinese Medicine. Rats were adaptively fed for 1 week. All rats were randomly and equally divided into two groups, including a control group and a TSW decoction group. TSW decoction was administered to the stomach for 1 week. All the animal experiments and protocols were approved by the local ethics committee.

2.2. Preparation of TSW-Containing Serum. TSW decoction was prepared using Semen Persicae, Flos Carthami, Angelica sinensis, Radix Paeoniae Alba, Rhizoma Chuanxiong, and Radix Rehmanniae Praeparata at fixed proportions as previously described [25]. TSW decoction was extracted as described and concentrated to $1.13 \mathrm{~g} / \mathrm{mL}$ [25]. Ten male SD rats were administered TSW decoction according to the following formula: rat dose $(\mathrm{g} / \mathrm{kg})=6.25 \times$ [adult dose $(\mathrm{g}) /$ adult body mass $(60 \mathrm{~kg})] \times 3$. In the TSW decoction group, rats were administered a dose of approximately $25 \mathrm{~g} / \mathrm{kg}$ TSW-containing serum twice a day for 1 week, intragastrically. The remaining rats in the blank group were given the same volume of normal saline for 1 week. After $2 \mathrm{~h}$ of gavage, they were treated with $40 \mathrm{mg} / \mathrm{kg}$ pentobarbital. The blood was collected from the abdominal aorta, and $5 \mathrm{~mL}$ blood was collected and left at $4^{\circ} \mathrm{C}$ overnight. Following centrifugation, the serum was collected, sterilised by filtration using a $0.22 \mu \mathrm{M}$ filter, inactivated at $56^{\circ} \mathrm{C}$, and stored at $-80^{\circ} \mathrm{C}$.

\subsection{Liquid Chromatography-Mass Spectrometry (LC-MS).} TSW decoction $(100 \mu \mathrm{L})$ was dissolved in $900 \mu \mathrm{L}$ deionized water. The mixed solution was then ultrasonicated in an ice bath for 15 minutes. Finally, the mixed solution was centrifuged at $12,000 \mathrm{rpm}$ for $10 \mathrm{~min}$. The supernatant was filtered through a $0.45 \mu \mathrm{m}$ membrane. The collected samples were analyzed by LC-MS (UHPLC: Nexera UHPLC LC-30A, Shimadzu; MASS: TripleTOF5600+, AB SCIEX $\left.{ }^{\mathrm{TM}}\right)$, using a Shimadzu InertSustain C18 column $(100 \times 2.1 \mathrm{~mm}, 2 \mu \mathrm{m})$. Mobile phases A and B for the LC were acetonitrile and $0.1 \%$ $\mathrm{CH}_{3} \mathrm{COOH}-\mathrm{H}_{2} \mathrm{O}$, respectively. The column temperature was $35^{\circ} \mathrm{C}$, and the flow rate was $0.3 \mathrm{~mL} / \mathrm{min}$. The LC-MS conditions are shown in Table 1. Electrospray ionization (ESI) positive ion and negative ion modes were used for detection. The ESI source conditions included ion source gas 1 (Gas 1, $50 \mathrm{psi}$ ), ion source gas 2 (Gas 2, $50 \mathrm{psi}$ ), and curtain gas (CUR, $25 \mathrm{psi}$ ). The source temperature was $500^{\circ} \mathrm{C}$ (positive ion) and $450^{\circ} \mathrm{C}$ (negative ion). Ion Sapary Voltage Floating (ISVF) was 5,500 V (positive ion) and 4,400 V (negative ion). The TOF MS scan range was $100-1200 \mathrm{Da}$. The product ion scan range was $50-1,000 \mathrm{Da}$. The TOF MS scan accumulation time was $0.2 \mathrm{~s}$, and the product ion scan accumulation time was $0.01 \mathrm{~s}$. Information-dependent acquisition was used for the secondary MS using a high sensitivity mode. The declustering potential was $\pm 60 \mathrm{~V}$, and the collision energy was $35 \pm 15 \mathrm{eV}$. The data were analyzed using Analysis Base File Converter and MS-DIAL 4.10 (Nature Methods, 12, 523-526, 2015) software. The extracted peak information was used to compare with the database and consisted of MassBank, ReSpect, and GNPS (14,951 records in total). In general, a total score greater than 80 was significant. The results are shown in Figure S1 and Table S1.

2.4. Isolation, Identification, and Culture of RAEC. The preparation of RAEC was based on isolation, identification, and culture previously described [2]. Briefly, dissection scissors were used to open the abdomen from the midline, and the abdominal aorta was exposed after blood perfusion. The aorta was stripped and digested to collect RAEC. The cells were collected, separated, and placed in a Petri dish, and the morphology of the cells was observed with an inverted fluorescence microscope. 
TABLE 1: LC conditions.

\begin{tabular}{lc}
\hline Time $(\min )$ & Parameter \\
\hline 0 & A: $2 \% ;$ B: $98 \%$ \\
2 & A: $4 \% ;$ B: $96 \%$ \\
4 & A: $10 \% ;$ B: $90 \%$ \\
7 & A: $14.5 \% ;$ B: $85.5 \%$ \\
8 & A: $14.5 \% ;$ B: $85.5 \%$ \\
12 & A: $23 \% ;$ B: $77 \%$ \\
16 & A: $46 \% ;$ B: $54 \%$ \\
26 & A: $100 \% ;$ B: $0 \%$ \\
28 & A: $100 \% ; B: 0 \%$ \\
28.5 & A: $2 \% ;$ B: $98 \%$ \\
\hline
\end{tabular}

2.5. Cell Treatment. RAEC were divided into five groups: a control group, a $2.5 \%$ TSW group, a $5 \%$ TSW group, a $10 \%$ TSW group, and a CAY10585 (an HIF-1 $\alpha$ inhibitor) group. The control group cells were treated with $10 \%$ blank serum ( $0 \%$ TSW) for $24 \mathrm{~h}$. The cells of the $2.5 \%, 5 \%$, and $10 \%$ TSW groups were treated with $2.5 \%$ TSW-containing serum, $5 \%$ TSW-containing serum, and 10\% TSW-containing serum, respectively, for $24 \mathrm{~h}$. We calculated the mixture of different volumes of TSW-containing serum and blank serum to ensure that the concentration of blank serum used in all groups was $10 \%$. The cells of the CAY 10585 group were treated with $10 \%$ TSW-containing serum in combination with $20 \mu \mathrm{M}$ CAY10585 (cat\# ab144422, Abcam, USA) for $24 \mathrm{~h}$. To further verify the effects of TSW-containing serum, the cells were divided into six groups consisting of the control group, the $2.5 \%$ TSW group, the 5\% TSW group, the 10\% TSW group, the CAY10585 group, and a KC7F2 group (another HIF inhibitor-1, cat\# S7946, Selleck, USA). The cells of the KC7F2 group were treated the same as that of the CAY10585 group.

2.6. Transwell Assay. A transwell chamber migration assay was used to examine the effects of TSW-containing serum and an HIF- $1 \alpha$ inhibitor on cell migration. The cells were seeded into 24 -well plates at $5 \times 10^{4}$ cells/well. The cells were treated as described above. After treatment for $48 \mathrm{~h}$, the cells at the bottom of the chamber were fixed with paraformaldehyde and then stained with $0.1 \%$ crystal violet. The migrated cells were observed and quantified with an inverted microscope. At least 5 different fields with more than 200 cells were counted, and the images were acquired.

2.7. MTT Assay. The MTT assay was used to detect the effects of TSW-containing serum on cell viability. Briefly, the cells were plated into 96-well plates at 3,000 cells/well and cultured for $24 \mathrm{~h}$. Then, $100 \mu \mathrm{L}$ MTT solution (Cat\#: M2128, Sigma, USA) was added and incubated for another $4 \mathrm{~h}$. The solution was aspirated, and $100 \mu \mathrm{L}$ DMSO was added to each well. The absorbance was measured using a microplate reader at a wavelength of $490 \mathrm{~nm}$.

2.8. Wound-Healing Assay. The cells were plated into 24well plates and then treated with TSW-containing serum in the presence or absence of an HIF- $1 \alpha$ inhibitor as described previously. Plates containing different groups of cells were scratched with a $20 \mu \mathrm{L}$ pipette to create a wound. The cells were washed with PBS buffer to remove the debris. Images were acquired at 0,24 , and $48 \mathrm{~h}$ after treatment to assess wound healing at 3 random locations. The wound area for each group was quantified.

2.9. Tube Formation Assay. Ninety-six-well plates were coated with $50 \mu \mathrm{L}$ Matrigel gel per well. The cells were then seeded into the 96-well plates at a density of $1.5 \times 10^{4}$ cells/ well. The cells were then treated with TSW-containing serum in the presence or absence of an HIF- $1 \alpha$ inhibitor as described previously. After incubation for $6 \mathrm{~h}$, tube formation was checked, and images were acquired with an inverted microscope. Images from at least 5 different fields were acquired using Image-Pro Plus 7.1 software to calculate the length of the lumen.

2.10. Quantitative Real-Time PCR ( $q R T-P C R)$. Total RNA was extracted from treated cells and then reverse-transcribed into cDNA using reverse transcriptase (Cat\#: K1622, Thermo Fisher, USA). The relative expression levels of HIF$1 \alpha$, VEGF, and VHL were measured by qRT-PCR using a $\mathrm{T}_{100}{ }^{\mathrm{TM}}$ thermal cycler. GAPDH was used as endogenous control, and the relative expression of the target genes was calculated using the $2^{-\Delta \Delta \mathrm{Ct}}$ method. The primers used for qRT-PCR are listed in Table 2.

2.11. Western Blot Analysis. Total protein was prepared from cells using lysis buffer (P0013, Beyotime Bio, China). Then, $30 \mu \mathrm{g}$ of protein from each group was separated by sodium dodecyl sulfate-polyacrylamide (SDS-PAGE) gels. Next, they were transferred onto polyvinylidene difluoride membranes (Millipore, USA). After blocking with 5\% fat-free milk in PBS buffer, the membranes were incubated with anti-HIF$1 \alpha$ (Abcam, USA), VEGF (Abcam, USA), VHL (Abcam, USA), and $\beta$-actin (Proteintech, USA) antibodies overnight at $4^{\circ} \mathrm{C}$. The membranes were then washed four times with PBST buffer for $5 \mathrm{~min}$ each time. The membranes were incubated with secondary antibodies for $1 \mathrm{~h}$ at room temperature. After washing with PBST buffer, protein bands were detected using enhanced chemiluminescence (Advans Group). The quantification of protein expression was done using ImageJ software (National Institutes of Health).

2.12. Statistical Analysis. All data were presented as the mean \pm standard deviation from at least three independent experiments. The results were analyzed using GraphPad Prism 8 software (GraphPad Prism Software Inc., San Diego, USA). The statistical difference among groups was examined using a one-way ANOVA test, followed by Tukey's post hoc test. $P<0.05$ was considered statistically significant.

\section{Results}

3.1. TSW Decoction Promoted Migration, whereas an HIF-1 $\alpha$ Inhibitor Reversed the Effects. TSW decoction was prepared from Semen Persicae, Flos Carthami, Angelica Sinensis, Radix 
TABle 2: Primer sequences.

\begin{tabular}{lrr}
\hline Name & Forward primer & Reverse primer \\
\hline HIF- $1 \alpha$ & ACGTTCCTTCGATCAGTTGTCACC & GGCAGTGGTAGTGGTGGCATTAG \\
VHL & TTTGTGCCATCTCTCAATGTTG & GGCATCGCTCTTTCAGAGTATA \\
VEGF & ATCGAGTACATCTTCAAGCCAT & GTGAGGTTTGATCCGCATAATC \\
GAPDH & AGTCCACTGGCGTCTTCAC & GAGGCATTGCTGATGATCTTGA \\
\hline
\end{tabular}

Paeoniae Alba, Rhizoma Chuanxiong, and Radix Rehmanniae Praeparata in fixed proportions as previously described [25]. To identify the specific components, we performed LCMS experiments. The results indicated that TSW decoction primarily contained isomaltulose, choline, D-gluconic acid, L-pipecolic acid, hypotaurine, albiflorin, and tryptophan (Figure S1 and Table S1). The LS-MS results showed that TWS decoction contained ferulic acid and amygdalin, and their relative peak areas were $1,562,890$ and 33,370 , respectively. Previous studies indicated that the main active ingredients of TSW decoction were ferulic acid, paeoniflorin, amygdalin, hydroxysafflor yellow A, catalpol, and gallic acid $[26,27]$. Therefore, we speculated that the main active components of TSW decoction were ferulic acid and amygdalin. Next, the rats were gavaged by TSW decoction and the acquired TSW-containing serum was used to treat RAEC.

RAEC were isolated from rats. To investigate the effects of TSW decoction on cell migration, the transwell assay was performed. The results indicated that TSW-containing serum enhanced the migration of cells in a dose-dependent manner (Figures 1(a) and 1(b)). After the fracture, HIF-1 $\alpha$ played an essential role in regulating hypoxia [28]. Therefore, we evaluated the effects of an HIF- $1 \alpha$ inhibitor on TSW-containing serum-promoted migration. The results demonstrated that $10 \%$ TSW-containing serum-induced migration was blocked by HIF inhibitor treatment of the cells (Figures 1(a) and 1(b)). The cells were treated with different concentrations of TSW-containing serum, and the MTT assay measured cell viability. The results indicated that $5 \%$ and $10 \%$ TSW-containing serum significantly increased the viability of the cells compared with the control group, suggesting a therapeutic effect in cells after a fracture. In contrast, CAY10585 and KC7F2 decreased the viability of the cells compared with the 10\% TSW group (Figure 1(c)).

3.2. TSW Decoction Expedited Migration, whereas HIF-1 $\alpha$ Inhibition Reversed the Effects. A wound-healing assay was carried out to examine the effects of TSW-containing serum on cell spreading. The results showed that the TSW-containing serum enhanced wound healing in a dose-dependent manner after the cells were treated for 24 and $48 \mathrm{~h}$. HIF- $1 \alpha$ inhibitor treatment reversed the effects of TSW-containing serum (Figure 2). These results suggested that TSW-containing serum regulated cell migration through HIF- $1 \alpha$ signaling.

3.3. TSW Decoction Accelerated Angiogenesis, whereas HIF-1 $\alpha$ Inhibition Decreased Angiogenesis. A tube formation assay was done to examine the effects of TSW-containing serum on RAEC angiogenesis. The results showed that TSWcontaining serum enhanced tube formation in a dose-dependent manner. HIF- $1 \alpha$ inhibitor treatment attenuated $10 \%$ TSW-containing serum-induced tube formation (Figure 3(a)). In addition, we measured the number of tubes and their length. The results showed that TSW-containing serum enhanced tube formation compared with the control group, whereas HIF-1 $\alpha$ inhibition attenuated the effects on tube formation (Figures 3(b) and 3(c)). These results suggested that TSW-containing serum regulated angiogenesis by increasing tube formation in cells through HIF- $1 \alpha$ signaling.

\subsection{TSW-Containing Serum Regulated the HIF-1 $\alpha / V E G F /$} VHL Signaling Pathway, whereas HIF-1 $\alpha$ Inhibition Reversed the Effects. To understand the role of HIF-1 $\alpha$ signaling in the angiogenesis of RAEC, we measured the expression of HIF$1 \alpha$, VEGF, and VHL mRNA. qRT-PCR results showed that the expression of HIF- $1 \alpha$ and VEGF were increased, whereas VHL expression was decreased, and these effects were reversed with HIF-1 $\alpha$ inhibitors (Figures 4(a) and 4(b)). Next, we measured the levels of HIF- $1 \alpha$, VEGF, and VHL protein by western blot analysis. The results showed that the levels of HIF- $1 \alpha$ and VEGF protein were elevated, whereas VHL expression was suppressed. HIF- $1 \alpha$ inhibitors attenuated these effects (Figures $4(\mathrm{c})$ and $4(\mathrm{~d})$ ). Altogether, the results demonstrated that TSW-containing serum regulated angiogenesis by activating the HIF- $1 \alpha / \mathrm{VEGF} / \mathrm{VHL}$ signaling pathway.

\section{Discussion}

Previous studies have shown that TCM promotes blood circulation, removes blood stasis, accelerates bone fracture healing and angiogenesis in injured areas, and improves blood circulation at the injured site [17-20]. Bone healing usually involves four steps: (1) hematoma formation; (2) soft callus formation, including the proliferation of vascular endothelial cells; (3) hard callus formation, and (4) threedimensional structure reconstruction of the lumen $[29,30]$. In the early stage of bone fracture, local bleeding and hematoma occur [31]. Along with the absorption and mechanization of the hematoma, an exudate is produced. This exudate promotes the secretion of vascular proliferation regulators, thereby accelerating the sprouting of micro blood vessels, which differentiate into blood vessels [30, 31]. Angioplasty is the formation of tubules consisting of vascular endothelial cells. After the tubules are formed, they are interwoven into a network with one another and penetrate the membrane to promote the growth of fractured ends and induce bone structure remodeling [6]. Therefore, detection 


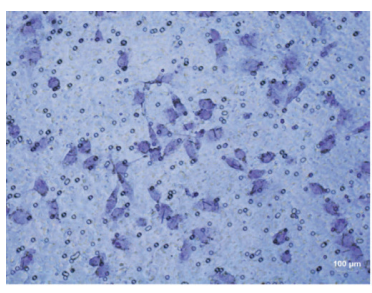

Control

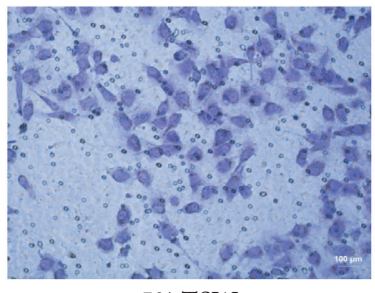

$5 \%$ TSW

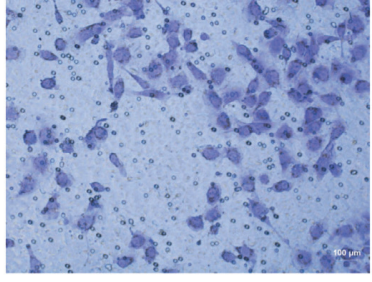

$2.5 \%$ TSW

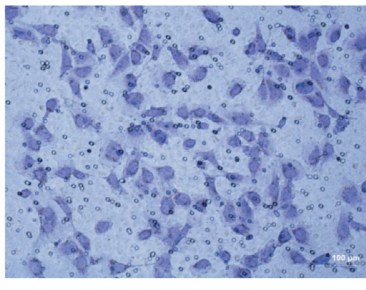

$10 \%$ TSW

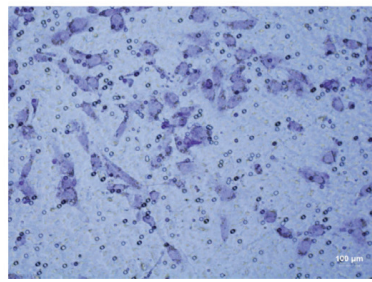

HIF inhibitor

(a)

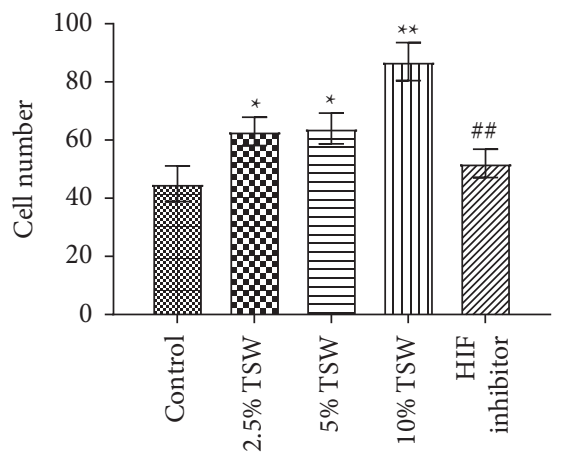

(b)

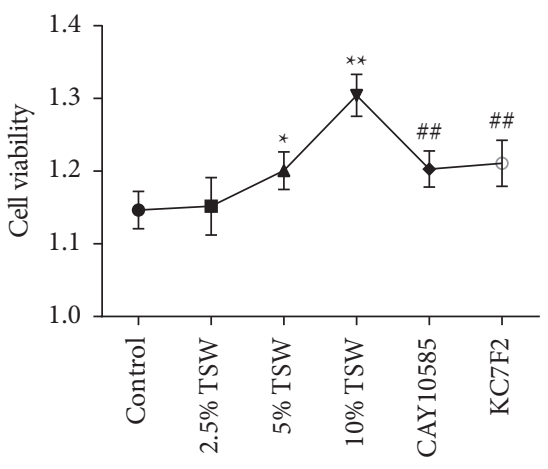

(c)

FIGURE 1: TSW decoction promoted migration, whereas an HIF-1 $\alpha$ inhibitor reversed the effects: (a) the migrated cells were detected using a transwell assay; (b) the migrated cells were counted and quantified; and (c) the cell viability was measured by MTT assay. ${ }^{* *} P<0.01$ and ${ }^{*} P<0.05$ versus the control group; ${ }^{\# \#} P<0.01$ and ${ }^{\#} P<0.05$ versus the $10 \%$ TSW group.

of vascular endothelial cell tubule formation as a marker of angiogenesis is also a prerequisite and basis for the healing process [6]. Our results showed that TSW-containing serum increased RAEC viability, indicating the efficacy of TSW decoction on RAEC growth. The results indicated that TSWcontaining serum enhanced the angiogenesis of RAEC by increasing the migration, spreading, and tube formation. Angiogenesis is a double-edged sword. In cancer, angiogenesis often promotes the growth of cancer cells [32, 33]. However, in wound healing, angiogenesis is often beneficial [34-36]. For example, blue-green microalga Spirulina platensis and collagen could elevate the expression of angiogenesis-related factors bFGF and VEGF to promote wound healing [34, 35]. Adipose-derived stem cells increased diabetic wound healing by stimulating the release of angiogenic factors [36]. In our study, angiogenesis was increased after TSW-containing serum treatment. Angiogenesis might further have beneficial effects on fracture healing. We speculated that TSW might improve blood circulation at the injured site and further induce bone structure remodeling.

After the fracture, periosteal and vascular damage occurs at the fracture site, and the local blood supply is reduced or even interrupted, resulting in hypoxia. Bone tissue in a hypoxic environment can activate HIF- $1 \alpha$, thereby activating the HIF/VEGF signaling pathway, which participates in the vascular reconstruction of fracture ends and accelerates the fracture healing process $[37,38]$. Next, we explored the underlying mechanism involved in TSWcontaining serum-induced endothelial cell angiogenesis. Interestingly, our results showed that an HIF- $1 \alpha$ inhibitor 


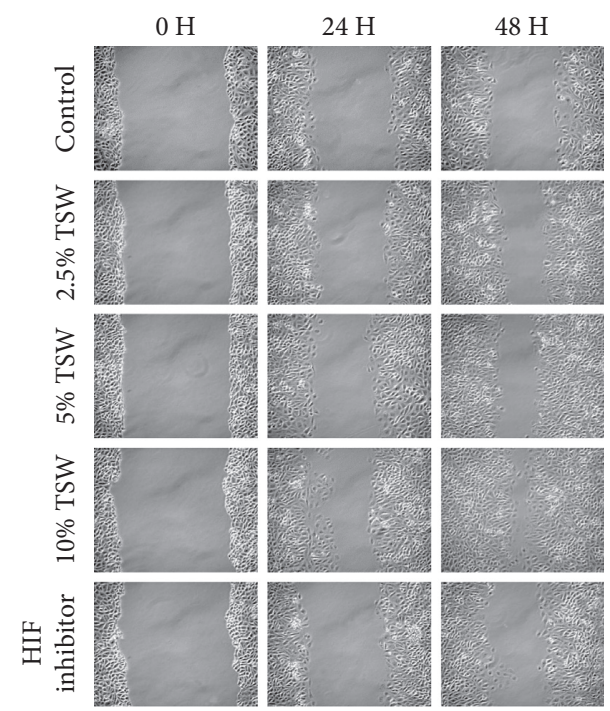

(a)

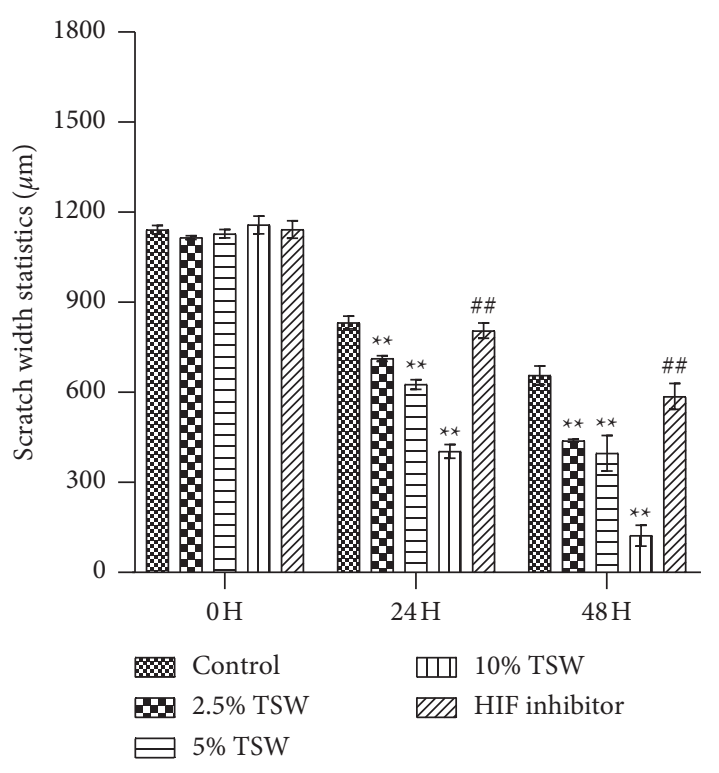

(b)

FIGURE 2: TSW decoction expedited migration, whereas HIF- $1 \alpha$ inhibition reversed the effects: (a) migrated cells were detected using a wound-healing assay and (b) the wound area in each group was calculated. ${ }^{* *} P<0.01$ versus the control group and ${ }^{\# \#} P<0.01$ versus the $10 \%$ TSW group.

dramatically attenuated TSW-containing serum-induced angiogenesis by inhibiting the migration, spreading, and tube formation of RAEC, indicating the involvement of HIF$1 \alpha$ signaling. Previous studies showed that HIF- $1 \alpha$ and VEGF expression are both upregulated during the healing process $[14,39]$. In addition, the expression of HIF- $1 \alpha$ mRNA is regulated by its upstream modulator VHL, which can induce the proteasome-dependent degradation of HIF$1 \alpha$ [39]. Recent studies have shown that the enhancement of angiogenesis and bone consolidation of some drugs during the process of osteogenesis may be related to the HIF-1 $\alpha /$ VEGF/VHL signaling pathway $[40,41]$. Our research showed that the TSW-containing serum enhanced angiogenesis by activating the HIF-1 $\alpha / \mathrm{VEGF} / \mathrm{VHL}$ signaling pathway. Moreover, the inhibition of HIF- $1 \alpha$ expression reversed the effects of TSW decoction and inhibited the expression of HIF- $1 \alpha$ /VEGF.

Recent research showed that ferulic acid and amygdalin could promote bone fracture healing and stimulate blood circulation $[42,43]$. Amygdalin effectively alleviates the inflammatory response in carrageenan-induced arthritis of rats [44]. The LS-MS results showed that TWS decoction contained ferulic acid and amygdalin, which might be the main active components. However, we cannot be sure of all the active ingredients at work in the TWS decoction. This was one of our limitations. We hope to carry out further experimental studies in the future to elaborate on the key substances in the TWS decoction. TSW-containing serum was obtained directly by gavage animals with TSW decoction. Because the gastrointestinal system of animals can selectively absorb and metabolize the components contained in TCM, the active components are probably similar, but their percentage may be different. The essence of the active ingredients was still from TSW decoction. We speculated that some of the main active ingredients of TSW decoction might be absorbed but could be detected in serum. For example, Liu et al. reported that songorine, benzoylhypaconitine, benzoylmesaconitine, and six other active ingredients were detected in the Yougui pill (YGP). Six of these components were also detected in the animal plasma of oral YGP in experimental autoimmune encephalomyelitis model rats. In addition, in vitro studies showed that YGP-containing serum and YGP extract had the same effects on the CREB/GAP-43 pathway [45]. Therefore, we speculated that TSW-containing serum might contain some effective components of TSW decoction. Because the serum compositions were too complex and the interference effects were too strong, the main components were detected in the decoction by LC-MS in many studies related to TCM. For example, the Actinidia chinensis Planch. and yam components were analyzed in gastric cancer and cartilage differentiation studies instead of the related drug-containing serum [46, 47]. Due to fund limitations, we did not research in depth on TSW-containing serum and TSW decoction components, which was a limitation of our research. However, we used blank serum as a control to eliminate the interference of serum. Essentially, the effects on the cells still come from the active ingredient of TSW decoction. We hope to carry out further experimental studies in the future to elaborate on the key substances and their in-depth mechanism of action in TSW decoction. 

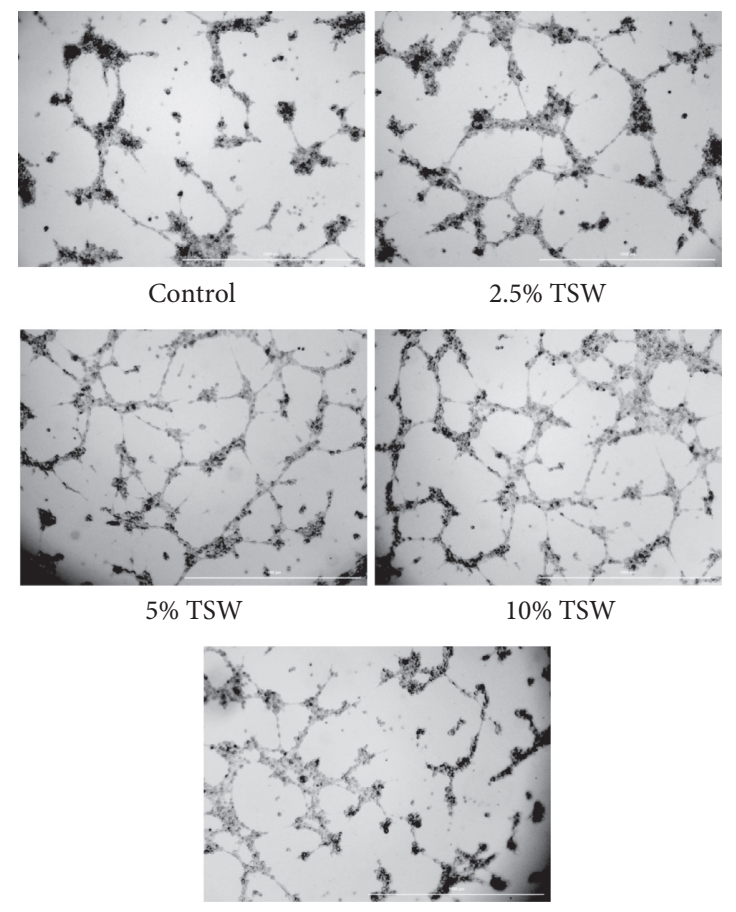

HIF inhibitor

(a)

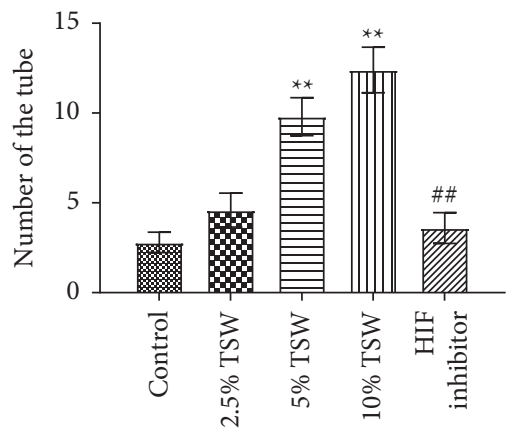

(b)

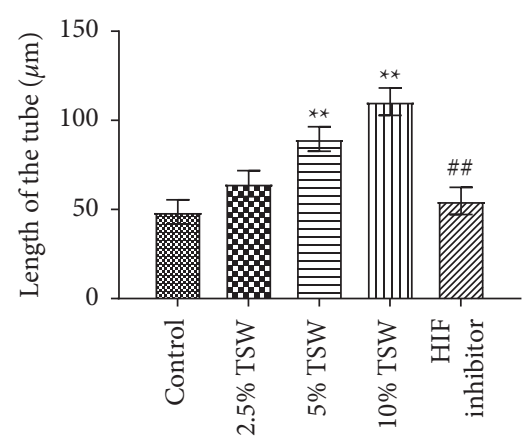

(c)

FIGURE 3: TSW decoction accelerated angiogenesis, whereas HIF- $1 \alpha$ inhibition decreased angiogenesis: (a) angiogenesis was analyzed using an angiogenesis experiment, (b) the number of tubes in each group was quantified and analyzed, and (c) the length of the tube in each group was quantified. ${ }^{* *} P<0.01$ versus the control group and ${ }^{\# \#} P<0.01$ versus the $10 \%$ TSW group.

Based on the below reasons, TSW-containing serum was used for in vitro experiments. In vitro experiments are highly controlled and can reveal deeper mechanisms of action at the cellular, molecular, and gene levels. As is known to all, TCM is composed of complex and diverse chemical components. Their extracts contain both active components and a large number of ineffective components, some of which can affect and interfere with the in vitro experiments without specificity. The gastrointestinal system of the body can selectively absorb and metabolize the components contained in TCM so as to exert real pharmacological effects. Therefore, TCM decoction and pill cannot be directly added to in vitro cell culture. Many studies have shown that drug-containing rat serum is often used in vitro experiments [46-48]. In addition, the drug-containing rat serum has a good effect in simulated in vitro experiments. For example, the inhibition of Actinidia chinensis Planch. serum on gastric cancer and the effects of yam-containing serum on the chondrogenic differentiation have been reported $[46,48]$. Serum pharmacology is a scientific analysis method for extracting serum-containing drugs from animals taken TCM orally $[46,49,50]$.

The innovation of our article was to use TSW decoction to act on primary RAEC and study its promotion of angiogenesis and possible pathways. As far as we know, we were the first to demonstrate that the TSW-containing serum enhanced angiogenesis of RAEC by enhancing the RAEC viability and migration. LC-MS was used to analyze all the components of TSW decoction and combined with previous studies. We further studied its possible mechanism of action and blocked experiments with HIF- $1 \alpha$ inhibitors, confirming that TSW decoction may partially promote endothelial cell angiogenesis through the VEGF/VHL/HIF$1 \alpha$ signaling pathway. In future research, we will further 


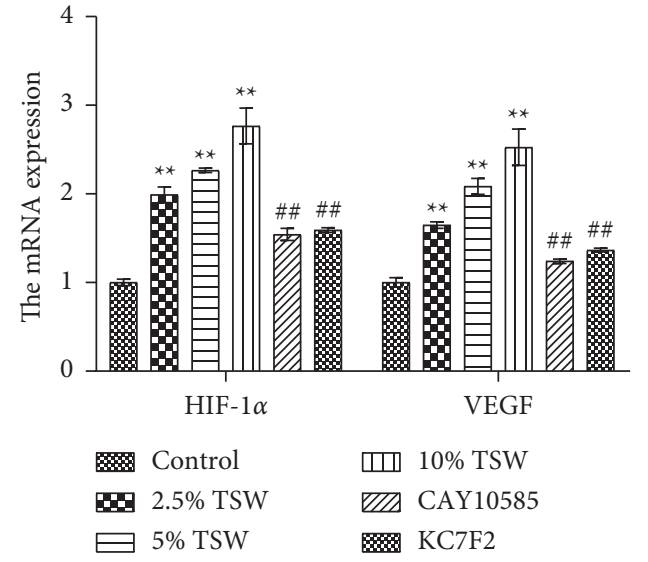

(a)

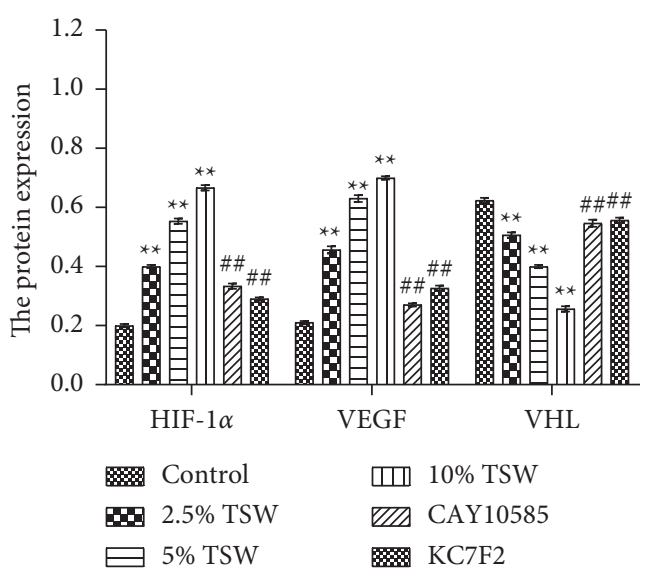

(c)
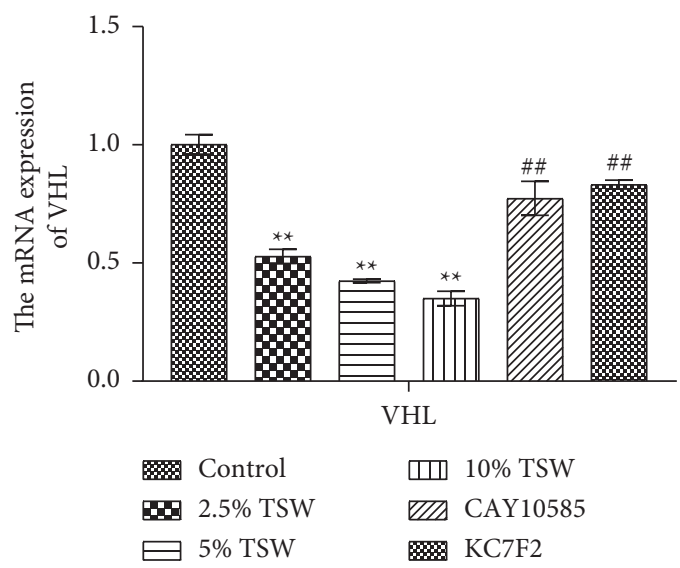

(b)

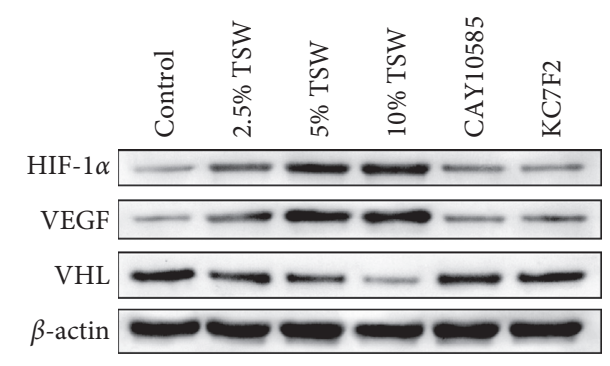

(d)

FIGURE 4: TSW decoction upregulated the HIF-1 $\alpha /$ VEGF/VHL signaling pathway, whereas HIF-1 $\alpha$ inhibitors reversed the effects: (a and $b$ ) the expression of HIF- $1 \alpha$, VEGF, and VHL mRNA was determined by qRT-PCR; (c) the expression of HIF- $1 \alpha$, VEGF, and VHL protein was quantified; and (d) the expression of HIF-1 $\alpha$, VEGF, and VHL protein was determined by western blot analysis. ${ }^{* *} P<0.01$ versus the control group and ${ }^{\# \#} P<0.01$ versus the $10 \%$ TSW group.

explore the action pathways of different components in TSW decoction. We will further study the mechanism of TSW decoction to develop effective drugs for the treatment of fracture patients.

\section{Conclusion}

Altogether, our results for the first time demonstrated that the TSW-containing serum enhanced angiogenesis of RAEC by enhancing the RAEC viability and migration. Mechanistic studies revealed that the effects of TSW-containing serum might be related to the VEGF/VHL/HIF- $1 \alpha$ signaling pathway in the RAEC angiogenesis.

\section{Abbreviations}

EPO: Erythropoietin

HRG: Hypoxia responsive genes

HIF- $1 \alpha$ : Hypoxia-inducible factor- $1 \alpha$

LC-MS: Liquid chromatography-mass spectrometry

RAEC: Rat aortic endothelial cells

SD: $\quad$ Sprague-Dawley
TCM: Traditional Chinese medicines

TSW: Taohong Siwu

VEGF: Vascular endothelial growth factor

VHL: Hippel-Lindau.

\section{Data Availability}

All the data generated or analyzed in this study are included in this manuscript, and the authors have confirmed the accuracy of the data.

\section{Ethical Approval}

All the animal experiments and protocols were approved by the local ethics committee.

\section{Consent}

The authors confirm that the manuscript has been read and approved by all named authors and that there are no other persons who satisfied the criteria for authorship but are not 
listed. The authors further confirm that the order of authors listed in the manuscript has been approved by all of them.

\section{Conflicts of Interest}

All the listed authors declare that they have no conflicts of interest.

\section{Authors' Contributions}

Zhi Tang performed the experiment and analyzed the data; Wangyang Li performed the experiment and prepared the manuscript; Hongzan Xie, Shengping Jiang, and Yunqing Pu performed the experiment; and Hui Xiong guided the experiment, reviewed, and edited the manuscript.

\section{Acknowledgments}

The authors would like to thankthe National Natural Science Foundation of China (81874478), Hunan Provincial Natural Science Foundation (2017JJ2256), and Xiangtan Science and Technology Bureau (SF-YB20181011) for their funding support.

\section{Supplementary Materials}

Figure S1: positive ion chromatogram and negative ion chromatogram of TSW Decoction were detected by LC-MS. Table S1: the components of TSW Decoction were detected by LC-MS. (Supplementary Materials)

\section{References}

[1] T. A. Einhorn and L. C. Gerstenfeld, "Fracture healing: mechanisms and interventions," Nature Reviews Rheumatology, vol. 11, no. 1, pp. 45-54, 2015.

[2] G. Gurel Pekozer, G. Torun Kose, and V. Hasirci, "Influence of co-culture on osteogenesis and angiogenesis of bone marrow mesenchymal stem cells and aortic endothelial cells," Microvascular Research, vol. 108, pp. 1-9, 2016.

[3] G. Walters, I. Pountos, and P. V. Giannoudis, "The cytokines and micro-environment of fracture haematoma: current evidence," Journal of Tissue Engineering and Regenerative Medicine, vol. 12, no. 3, pp. e1662-e1677, 2018.

[4] X. X. Zhu, X. Y. Miao, Y. P. Gong, B. Fu, and C. L. Li, "Isolation and culture of rat aortic endothelial cells in vitro: a novel approach without collagenase digestion," Journal of Cellular Biochemistry, vol. 120, no. 8, pp. 14127-14135, 2019.

[5] J. Zhou, J. H. Rogers, S. H. Lee et al., "Oral mucosa harbors a high frequency of endothelial cells: a novel postnatal cell source for angiogenic regeneration," Stem Cells and Development, vol. 26, no. 2, pp. 91-101, 2017.

[6] M. H. Lafage-Proust, B. Roche, M. Langer et al., "Assessment of bone vascularization and its role in bone remodeling," Bonekey Reports, vol. 4, p. 662, 2015.

[7] O. Hudlicka and M. D. Brown, "Adaptation of skeletal muscle microvasculature to increased or decreased blood flow: role of shear stress, nitric oxide and vascular endothelial growth factor," Journal of Vascular Research, vol. 46, no. 5, pp. 504-512, 2009.

[8] E. Muinos-López, P. Ripalda-Cemboráin, T. López-Martínez et al., "Hypoxia and reactive oxygen species homeostasis in mesenchymal progenitor cells define a molecular mechanism for fracture nonunion," Stem Cells, vol. 34, no. 9, pp. 2342-2353, 2016.

[9] M. Y. Koh, R. Lemos, X. Liu, and G. Powis, "The hypoxiaassociated factor switches cells from HIF- $1 \alpha$-to HIF- $2 \alpha$-dependent signaling promoting stem cell characteristics, aggressive tumor growth and invasion," Cancer Research, vol. 71, no. 11, pp. 4015-4027, 2011.

[10] M. H. Chen, Q.-X. Ren, W.-F. Yang, X.-L. Chen, C. Lu, and J. Sun, "Influences of HIF-lalpha on Bax/Bcl-2 and VEGF expressions in rats with spinal cord injury," International Journal of Clinical and Experimental Pathology, vol. 6, no. 11, pp. 2312-2322, 2013.

[11] B. L. Krock, N. Skuli, and M. C. Simon, "Hypoxia-induced angiogenesis: good and evil," Genes \& Cancer, vol. 2, no. 12, pp. 1117-1133, 2011.

[12] L. Chen, A. Endler, and F. Shibasaki, "Hypoxia and angiogenesis: regulation of hypoxia-inducible factors via novel binding factors," Experimental and Molecular Medicine, vol. 41, no. 12, pp. 849-857, 2009.

[13] E. Schipani, C. Maes, G. Carmeliet, and G. L. Semenza, "Regulation of osteogenesis-angiogenesis coupling by HIFs and VEGF," Journal of Bone and Mineral Research, vol. 24, no. 8, pp. 1347-1353, 2009.

[14] E. B. Rankin, A. J. Giaccia, and E. Schipani, "A central role for hypoxic signaling in cartilage, bone, and hematopoiesis," Current Osteoporosis Reports, vol. 9, no. 2, pp. 46-52, 2011.

[15] S. Pal, K. P. Claffey, H. F. Dvorak, and D. Mukhopadhyay, "The von Hippel-Lindau gene product inhibits vascular permeability factor/vascular endothelial growth factor expression in renal cell carcinoma by blocking protein kinase $\mathrm{C}$ pathways," Journal of Biological Chemistry, vol. 272, no. 44, pp. 27509-27512, 1997.

[16] T. Kamura, S. Sato, K. Iwai, M. Czyzyk-Krzeska, R. C. Conaway, and J. W. Conaway, "Activation of HIFlalpha ubiquitination by a reconstituted von Hippel-Lindau (VHL) tumor suppressor complex," Proceedings of the National Academy of Sciences, vol. 97, no. 19, pp. 10430-10435, 2000.

[17] C. Y. Tseng, C.-W. Huang, H.-C. Huang, and W.-C. Tseng, "Utilization pattern of traditional Chinese medicine among fracture patients: a Taiwan hospital-based cross-sectional study," Evidence-Based Complementary and Alternative Medicine, vol. 2018, Article ID 1706517, 9 pages, 2018.

[18] T.-P. Hsueh and H. E. Chiu, "Traditional Chinese medicine speeds-up humerus fracture healing: two case reports," Complementary Therapies in Medicine, vol. 20, no. 6, pp. 431-433, 2012.

[19] W. S. Siu, H. T. Shiu, C. H. Ko et al., "Integrative approach to facilitate fracture healing: topical Chinese herbal paste with oral strontium ranelate," Evidence-Based Complementary and Alternative Medicine, vol. 2017, Article ID 9795806, 11 pages, 2017.

[20] X.-L. Wang, X.-P. Zhu, D.-X. Ji et al., "Beneficial effect of traditional Chinese medicine fumigation "bone-healing powder" in postoperative pain and recovery of neurological function of traumatic thoracolumbar spine fractures: a casecontrol study," Medicine, vol. 97, no. 35, Article ID e11983, 2018.

[21] C.-S. Zheng, X.-J. Xu, H.-Z. Ye et al., "Network pharmacology-based prediction of the multi-target capabilities of the compounds in Taohong Siwu decoction, and their application in osteoarthritis," Experimental and Therapeutic Medicine, vol. 6, no. 1, pp. 125-132, 2013. 
[22] S. Zhu, Y. Song, X. Chen, and W. Qian, "Traditional Chinese and western medicine for the prevention of deep venous thrombosis after lower extremity orthopedic surgery: a metaanalysis of randomized controlled trials," Journal of Orthopaedic Surgery and Research, vol. 13, no. 1, p. 79, 2018.

[23] D. Yin, Z. Liu, D. Peng et al., "Serum containing Tao-Hong-Si$\mathrm{Wu}$ decoction induces human endothelial cell VEGF production via PI3K/Akt-eNOS signaling," Evidence Based Complementary and Alternative Medicine, vol. 2013, Article ID 195158, 9 pages, 2013.

[24] J. Liang, D. K. Yin, B. K. Li et al., "[Effect of Taohong Siwu decoction on angiogenesis of medicine-induced incompleteabortion in early pregnancy rats and expressions of Ang-1, Ang-2 and Tie-2]," Zhongguo Zhongyao Zazhi, vol. 38, no. 21, pp. 3731-3735, 2013.

[25] Z. R. Luo, H. Li, Z. X. Xiao et al., “Taohong siwu decoction exerts a beneficial effect on cardiac function by possibly improving the microenvironment and decreasing mitochondrial fission after myocardial infarction," Cardiology Research and Practice, vol. 2019, Article ID 5198278, 13 pages, 2019.

[26] P. Liu, J. A. Duan, G. Bai, and S. L. Su, "[Network pharmacology study on major active compounds of siwu decoction analogous formulae for treating primary dysmenorrhea of gynecology blood stasis syndrome]," Zhongguo Zhongyao Zazhi, vol. 39, no. 1, pp. 113-120, 2014.

[27] Y. Zhang, C. Zuo, L. Han et al., "Uterine metabolomics reveals protection of Taohong siwu decoction against abnormal uterine bleeding," Frontiers in Pharmacology, vol. 11, Article ID 507113, 2020.

[28] K. Hu, S. Babapoor-Farrokhran, M. Rodrigues et al., "Hypoxiainducible factor 1 upregulation of both VEGF and ANGPTL4 is required to promote the angiogenic phenotype in uveal melanoma," Oncotarget, vol. 7, no. 7, pp. 7816-7828, 2016.

[29] B. Beamer, C. Hettrich, and J. Lane, "Vascular endothelial growth factor: an essential component of angiogenesis and fracture healing," HSS Journal, vol. 6, no. 1, pp. 85-94, 2010.

[30] C. Lu, R. Marcucio, and T. Miclau, "Assessing angiogenesis during fracture healing," The Iowa Orthopaedic Journal, vol. 26, pp. 17-26, 2006.

[31] H. Schell, G. N. Duda, A. Peters, S. Tsitsilonis, K. A. Johnson, and K. Schmidt-Bleek, "The haematoma and its role in bone healing," Journal of Experimental Orthopaedics, vol. 4, no. 1, p. $5,2017$.

[32] D. Unterleuthner, P. Neuhold, K. Schwarz et al., "Cancerassociated fibroblast-derived WNT2 increases tumor angiogenesis in colon cancer," Angiogenesis, vol. 23, no. 2, pp. 159-177, 2020.

[33] X.-G. Wu, C.-F. Zhou, Y.-M. Zhang et al., "Cancer-derived exosomal miR-221-3p promotes angiogenesis by targeting THBS2 in cervical squamous cell carcinoma," Angiogenesis, vol. 22, no. 3, pp. 397-410, 2019.

[34] Z. I. Elbialy, D. H. Assar, A. Abdelnaby et al., "Healing potential of Spirulina platensis for skin wounds by modulating bFGF, VEGF, TGF- $\beta 1$ and $\alpha$-SMA genes expression targeting angiogenesis and scar tissue formation in the rat model," Biomedicine \& Pharmacotherapy, vol. 137, Article ID 111349, 2021.

[35] Z. I. Elbialy, A. Atiba, A. Abdelnaby et al., "Collagen extract obtained from Nile tilapia (Oreochromis niloticus L.) skin accelerates wound healing in rat model via up regulating VEGF, bFGF, and $\alpha$-SMA genes expression," BMC Veterinary Research, vol. 16, no. 1, p. 352, 2020.
[36] M. Gadelkarim, A. I. Abushouk, E. Ghanem, A. M. Hamaad, A. M. Saad, and M. M. Abdel-Daim, "Adipose-derived stem cells: effectiveness and advances in delivery in diabetic wound healing," Biomedicine \& Pharmacotherapy, vol. 107, pp. 625-633, 2018.

[37] M. Marenzana and T. R. Arnett, "The key role of the blood supply to bone," Bone Research, vol. 1, no. 3, pp. 203-215, 2013.

[38] P. J. Bouletreau, S. M. Warren, J. A. Spector et al., "Hypoxia and VEGF up-regulate BMP-2 mRNA and protein expression in microvascular endothelial cells: implications for fracture healing," Plastic and Reconstructive Surgery, vol. 109, no. 7, pp. 2384-2397, 2002.

[39] C. Wan, S. R. Gilbert, Y. Wang et al., "Activation of the hypoxia-inducible factor-1 pathway accelerates bone regeneration," Proceedings of the National Academy of Sciences, vol. 105, no. 2, pp. 686-691, 2008.

[40] Z. Tang, H. Xie, S. Jiang et al., "Safflower yellow promotes angiogenesis through $\mathrm{p}$-VHL/HIF- $1 \alpha$ /VEGF signaling pathway in the process of osteogenic differentiation," Biomedicine \& Pharmacotherapy, vol. 107, pp. 1736-1743, 2018.

[41] M. Hamushan, W. Cai, Y. Zhang et al., "High-purity magnesium pin enhances bone consolidation in distraction osteogenesis model through activation of the VHL/HIF- $1 \alpha /$ VEGF signaling," Journal of Biomaterials Applications, vol. 35, no. 2, pp. 224-236, 2020.

[42] K. Du, Z. Li, X. Fang, T. Cao, and Y. Xu, "Ferulic acid promotes osteogenesis of bone marrow-derived mesenchymal stem cells by inhibiting microRNA-340 to induce $\beta$-catenin expression through hypoxia," European Journal of Cell Biology, vol. 96, no. 6, pp. 496-503, 2017.

[43] J. Ying, Q. Ge, S. Hu et al., "Amygdalin promotes fracture healing through TGF- $\beta$ /smad signaling in mesenchymal stem cells," Stem Cells International, vol. 2020, Article ID 8811963, 13 pages, 2020.

[44] H. J. Hwang, H. J. Lee, C. J. Kim, I. Shim, and D. H. Hahm, "Inhibitory effect of amygdalin on lipopolysaccharide-inducible TNF-alpha and IL-1beta mRNA expression and carrageenan-induced rat arthritis," Journal of Microbiology and Biotechnology, vol. 18, no. 10, pp. 1641-1647, 2008.

[45] H. Liu, F. Qiu, X. Yang, H. Zhao, B. Bian, and L. Wang, "Pharmacokinetics of the Yougui pill in experimental autoimmune encephalomyelitis model rats and its pharmacological activity in vitro," Drug Design, Development and Therapy, vol. 13, pp. 2357-2370, 2019.

[46] Z. Gao, G. Deng, Y. Li et al., "Actinidia chinensis planch prevents proliferation and migration of gastric cancer associated with apoptosis, ferroptosis activation and mesenchymal phenotype suppression," Biomedicine \& Pharmacotherapy, vol. 126, Article ID 110092, 2020.

[47] Y. Li, Y. Li, Z. Zou, Y. Li, H. Xie, and H. Yang, "Yin Yang Gong Ji pill is an ancient formula with antitumor activity against hepatoma cells," Journal of Ethnopharmacology, vol. 248, Article ID 112267, 2020.

[48] R. Yang, R. Zhang, H. Deng et al., "Yam-containing serum promotes proliferation and chondrogenic differentiation of rabbit bone marrow mesenchymal stem cells and synthesis of glycosaminoglycan," Pharmacology, vol. 105, no. 7-8, pp. 377-385, 2020.

[49] X.-Z. Chen, Z.-Y. Cao, L.-M. Liao, Z.-Z. Liu, and J. Du, "Application of serum pharmacology in evaluating the antitumor effect of Fuzheng Yiliu decoction from Chinese medicine," Chinese Journal of Integrative Medicine, vol. 20, no. 6, pp. 450-455, 2014. 
[50] W. Bochu, Z. Liancai, and C. Qi, "Primary study on the application of serum pharmacology in Chinese traditional medicine," Colloids and Surfaces B: Biointerfaces, vol. 43, no. 3-4, pp. 194-197, 2005. 